\title{
Polydrug Use among IDUs in Tijuana, Mexico: Correlates of Methamphetamine Use and Route of Administration by Gender
}

\author{
Melanie L. Rusch, Remedios Lozada, Robin A. Pollini, \\ Alicia Vera, Thomas L. Patterson, Patricia Case, \\ and Stefanie A. Strathdee
}

\begin{abstract}
Tijuana is situated on the Mexico-USA border adjacent to San Diego, CA, on a major drug trafficking route. Increased methamphetamine trafficking in recent years has created a local consumption market. We examined factors associated with methamphetamine use and routes of administration by gender among injection drug users (IDUs). From 2006-2007, IDUs $\geq 18$ years old in Tijuana were recruited using respondent-driven sampling, interviewed, and tested for HIV, syphilis, and TB. Logistic regression was used to assess associations with methamphetamine use (past 6 months), stratified by gender. Among 1,056 participants, methamphetamine use was more commonly reported among females compared to males $(80 \%$ vs. $68 \%, p<0.01)$, particularly, methamphetamine smoking $(57 \%$ vs. $34 \%$; $p<0.01)$. Among females $(\mathrm{N}=$ 158), being aged $>35$ years (AOR, 0.2; 95\% CI, 0.1-0.6) was associated with methamphetamine use. Among males ( $N=898)$, being aged $>35$ years ( $A O R, 0.5 ; 95 \%$ CI, 0.3-0.6), homeless (AOR, 1.4 (0.9-2.2)), and ever reporting sex with another male (MSM; AOR, 1.9; 95\% CI, 1.4-2.7) were associated with methamphetamine use. Among males, a history of MSM was associated with injection, while sex trade and $>2$ casual sex partners were associated with multiple routes of administration. HIV was higher among both males and females reporting injection as the only route of methamphetamine administration. Methamphetamine use is highly prevalent among IDUs in Tijuana, especially among females. Routes of administration differed by gender and subgroup which has important implications for tailoring harm reduction interventions and drug abuse treatment.
\end{abstract}

KEYWORDS Methamphetamine, Injection drug use, Sexual risk, Mexico, Commercial sex work

\section{INTRODUCTION}

Methamphetamine has emerged as a major drug of abuse in the Western and MidWestern US states, Western Canada, Southeast Asia, and parts of Western and Eastern Europe. ${ }^{1-3}$ In North America and Europe, methamphetamine use has been closely associated with unprotected sex and increased risk of HIV and other sexually transmitted infections (STIs) in men who have sex with men (MSM), ${ }^{4,5}$ and

Rusch, Pollini, Vera, and Strathdee are with the Division of Global Public Health, Department of Medicine, University of California San Diego, La Jolla, CA, USA; Lozada is with the Patronato Pro-COMUSIDA, Tijuana, Mexico; Patterson is with the Department of Psychiatry, University of California San Diego, La Jolla, CA, USA; Case is with The Fenway Institute, Fenway Community Health, Boston, MA, USA.

Correspondence: Melanie L. Rusch, Division of Global Public Health, Department of Medicine, UCSD, 9500 Gilman Drive, mailstop 0507, La Jolla, CA 92093-0507, USA. (E-mail: mrusch@ucsd.edu) 
increasingly in heterosexual women. ${ }^{6-8}$ Among injection drug users (IDUs), methamphetamine use has been associated with higher levels of needle sharing and other high-risk injection behaviors. ${ }^{9,10}$

Gender differences have been reported in terms of prevalence of substance use, and motivations for initiation and continued use of various illicit drugs. ${ }^{11-13}$ Psychological co-morbidities, childhood abuse, substance use initiation with a sexual partner, progression to dependence, and treatment responsiveness have been shown to be higher among females for many substances of abuse, including methamphetamine. ${ }^{11,12,14}$ Although a recent review concluded that females tend to initiate substance use later than males, methamphetamine use was an exception, with earlier initiation among females compared to males. ${ }^{11}$ While men tend to have higher prevalence of substance use, studies from the US have shown comparable prevalence of methamphetamine use in men and women. ${ }^{15}$ Female methamphetamine users have also been shown to have lower substitution of other substances when methamphetamine is not available and to have higher levels of dependence compared to men. ${ }^{12}$

Compared to other substances including cocaine, methamphetamine is more often linked to sexual motivations for use, positive sexual associations (drive, performance, pleasure), and increased sexual activity, including riskier behaviors such as increased casual partners and decreased condom use. ${ }^{6,7,9,12,16,17}$ In addition there are a number of studies among MSM populations linking methamphetamine use to sexual motivations and sexual environments such as bathhouses and circuit parties. ${ }^{18-20}$ Likewise, recent studies have shown that women who have sex with women (WSW) also report higher prevalence of substance use, including methamphetamine. ${ }^{21-23}$ While higher sexual risk has been reported among both males and females, differences in motivations and sexual perceptions have been noted. For example, several studies have found females more often indicate energy, weight-loss, and mood as reasons for using methamphetamine. ${ }^{7,11,12}$ Psychiatric co-morbidities are high among methamphetamine users in general; however, depression has been shown to be especially high among female users, who are also more likely to report using methamphetamine to cope with mood. ${ }^{11,24}$ These motivations for use could be particularly important to consider among female sex workers (FSW) who may be more likely to experience depression, to be concerned about weight and to require energy for work. Sexual motivations for methamphetamine use are also reported among both male and female users, although typically more so among males. ${ }^{12}$ In one study comparing sexual associations with stimulants, both males and females indicated increased positive sexual associations with methamphetamine compared to cocaine; however, an overall higher proportion of males reported sexual effects as compared to females. ${ }^{16}$

The route of drug administration may be linked to the level of dependence, the context of use and the types and number of illicit substances used. In general, oral, intranasal, and anal absorption routes of administration are reported more often by occasional users and tend to result in lower bioavailability and less severe dependence. ${ }^{25,26}$ Injection of methamphetamine has been reported to be associated with higher levels of dependence; however, more chaotic patterns of use have been found among methamphetamine smokers. ${ }^{25,27,28}$ A study comparing injectors, smokers, and those who both smoke and inject reported comparable levels of sexual risk, psychoses and psychological distress, and involvement in drug-related crime; although those who both smoke and inject tend to exhibit the highest risks. ${ }^{25}$ Studies among IDUs have shown that injection of methamphetamine is associated not only 
with increased sexual risk, including higher numbers of partners and increased involvement in sex trade, but also with increased needle sharing and failure to clean used needles. ${ }^{9}$ Most studies that examine methamphetamine use and its relation to HIV/STI risk behaviors focus on populations that are primarily stimulant users. To our knowledge, studies are lacking that examine gender differences in risk behaviors by route of administration.

Tijuana, situated on the Mexico-USA border adjacent from San Diego, CA, is on a major drug trafficking route. Since the 1970 s, up to $50 \%$ of the cocaine coming from South America has entered the USA through the USA-Mexico land border; ${ }^{1,29}$ Mexico also produces $30 \%$ of all heroin entering the USA. ${ }^{30}$ More recently, crackdowns on methamphetamine production in the USA have led to increased demand on Mexican producers, who are now estimated to supply the majority of methamphetamine distributed in the USA. ${ }^{29}$ Increased production of methamphetamine in Mexico has led to the emergence of a local methamphetamine consumption market in Tijuana. ${ }^{29,31}$ In a series of in-depth interviews conducted in 2004, members of our binational research team found evidence to suggest that methamphetamine had already become a major drug of abuse in Tijuana, and was emerging as a new drug of abuse among IDUs in Ciudad Juarez. ${ }^{32}$

The present analysis sought to assess differences in the demographics and risk behaviors associated with methamphetamine use among a cohort of IDU in Tijuana, Mexico, with a high prevalence of poly substance use. We hypothesized that the prevalence of methamphetamine use would be comparable among male and female IDU, but that characteristics and risk behaviors associated with specific routes administration would differ, reflecting particular motivations and contexts of use. Exploring differences by gender and route of administration could uncover differences in the context of substance use and sexual risk that may be useful in planning interventions tailored to the needs of male and female IDUs.

\section{METHODS}

\section{Study Population}

During 2006-2007, 1,056 IDUs were recruited in Tijuana using respondent-driven sampling (RDS), as previously described. ${ }^{33}$ RDS is a type of snowball sampling which includes the collection of information about recruitment networks in order to calculate weights to reduce bias in population estimates which may occur through oversampling of homogenous networks. ${ }^{34}$ A diverse group of 32 seeds, heterogeneous by age (median, 38 ; range, $22-70)$, gender (20\% female), and neighborhood (60\% from areas outside of the Zona Roja, the main area frequented by injectors), were initially invited to participate and given three coupons to refer peers to the study. Individuals recruited by the initial seeds were subsequently given coupons to recruit members of their own social networks. Participants were paid a small amount (approximately 5 USD) for successful recruitment of eligible members of their social network. Eligibility criteria included being 18 years of age or older, injection drug use in the past 6 months (verified through inspection of stigmata, or 'track marks'), ability to speak Spanish or English, and having no plans to move from Tijuana over the next 18 months. Interviews were conducted by indigenous outreach workers at both a fixed storefront location and at various neighborhood locations using a modified mobile recreational vehicle. Participants were reimbursed the equivalent of 20 USD for their participation in the survey and biological 
sampling procedures. The study was approved by the Institutional Review Board of the University of California, San Diego and the Ethics Board of the Tijuana General Hospital.

\section{Data Collection}

The survey included sociodemographics, questions about the participants' social and injection networks, substances used, frequency of use and route of administration (i.e., smoking, snorting, or injection), sexual behaviors, migration and immigration, as well as incarceration and institutionalization. Among males, MSM was defined as ever reporting sexual activity with another male. Sex work was defined as trading sex for money, drugs, or other goods. Participants were asked a series of questions about their substance use, with specific reference to the type of drug and route of administration. For example, the interviewer would ask if they had "ever smoked methamphetamine (crystal) by itself?", followed by a question about their age at first use and the frequency of use in the past 6 months. Participants were asked separately about injecting methamphetamine by itself and in combination with cocaine, heroin, or other drugs.

\section{HIV and STI Testing}

Participants underwent a blood draw for HIV and syphilis antibody testing. HIV serostatus was assessed using the Determine Rapid HIV Antibody Test (Abbott Pharmaceuticals, Boston, MA), with confirmatory testing on all reactive samples using the HIV-1 enzyme immunoassay and immunofluorescence assay. Both qualitative and quantitative RPR (Macro-Vue; Becton Dickinson, Cockeysville, MD) and confirmatory Treponema pallidum particle agglutination (TPPA; Fujirebio, Wilmington, DE) tests were performed for detection of syphilis antibody. Confirmatory testing of all specimens was done through the San Diego County Health Department. Participants with positive test results were referred to free health care through the Tijuana municipal health clinic.

\section{Statistical Analysis}

The main outcome of interest was methamphetamine use in the past 6 months. A descriptive profile of methamphetamine users was assessed including age, gender, income, homelessness, involvement in sex trade, number of casual sex partners, and among men, sexual activity with other men (MSM) and among women, and sexual activity with other women (WSW). Logistic regression was used to assess factors independently associated with recent methamphetamine use, stratified by gender. RDS-adjusted models were first run, using weights calculated from RDSAT software. ${ }^{35}$ These methods have been reported elsewhere. ${ }^{33}$ Briefly, individualized weights are calculated based on the outcome of interest, accounting for non-random recruitment chains. However, as estimates were not altered in the weighted models, unweighted models are presented here. Models were adjusted for potential clustering based on recruiter networks. Variables with a $p$ value $<0.10$ in univariate models were assessed in multivariate models and retained in the final adjusted model. As there was an expected association of younger age with methamphetamine use and the association of age with sexual behaviors, interactions were assessed for age and number of casual partners, sex trade, and among males, MSM. Finally, exploratory univariate logistic regression models were used to assess associations of the above risk variables with specific routes of methamphetamine administration. Routes of administration were categorized as mutually exclusive groups reporting injection 
only, smoking only, injection and smoking, injection and snorting, smoking and snorting, and all three routes (injection, smoking, and snorting).

\section{RESULTS}

\section{Participants}

Among 1,056 participants, 898 (85\%) were male and 158 (15\%) were female. The median age was 36 (interquartile range [IQR], 31-42) and over one quarter $(28.5 \%)$ were born in Tijuana. Less than half $(41.5 \%)$ had completed a high school education and over half $(56.5 \%)$ had a monthly income of less than 3,500 pesos (approximately 335 USD). Fourteen percent were homeless, and 36\% lived in the Zona Norte region of the city where the red light district (Zona Roja) is located. Female IDUs were significantly younger than their male counterparts [median age (IQR), $34(27,40)$ vs. $36(31,42)$, respectively], and were more likely to report completion of high school ( $47 \%$ vs. $40 \% ; p=0.097)$. Male IDUs were more likely to report a monthly income greater than 3,500 pesos $(45 \%$ vs. $35 \% ; p=0.017)$ and were more likely to report being homeless $(15 \%$ vs. $5 \% ; p=0.001)$. Male IDU were less likely to report any sexual activity $(28 \%$ vs. $58 \%$; $p<0.01)$. By gender, the proportions reporting specific partner types were similar (males, 14\% regular, $12 \%$ casual; females, $35 \%$ regular, $34 \%$ casual). Inconsistent condom use with regular partners was high for both male and female IDUs $(91 \%$ vs. $83 \%$; $p=0.15)$. With casual partners, inconsistent condom use was lower; however, males reported higher risk than females $(65 \%$ vs. $40 \%$; $p<0.01)$. The baseline HIV prevalence in the cohort was $4.5 \%$ (RDS-adjusted prevalence, $3.0 \%$ ), with a higher proportion of cases among female IDU [ $10.2 \%$ vs. $3.5 \%$ (RDS adjusted, $5.4 \%$ vs. $2.4 \%$ ); $p<0.01]$. The overall syphilis prevalence was $7.2 \%$ (RDS-adjusted prevalence, $5.1 \%$ ), again with a higher proportion of cases in females [16\% vs. $6 \%$ (RDS adjusted, $9.2 \%$ vs. $4.0 \%$ ); $p<0.01$ ].

\section{Substance Use and Route of Methamphetamine Administration by Gender}

Heroin was the most prevalent substance of abuse reported- $-89 \%, 30 \%, 19 \%$, and $<5 \%$ reported more than once daily injection of heroin, heroin mixed with methamphetamine, methamphetamine, and cocaine, respectively. Of those reporting any methamphetamine use, $98 \%$ also reported heroin use. The median number of different substances used was two (IQR, 2, 3) and did not differ by gender. While heroin and methamphetamine were the most common substances reported for daily use, use of other substances in the past 6 months included marijuana (37\%), cocaine $(21 \%)$, tranquilizers $(15 \%)$, barbiturates $(2.5 \%)$, and others $(1.1 \%)$.

Figure 1 illustrates the proportion of methamphetamine users overall and by route of administration comparing male and female IDUs. Females were more likely than males to report methamphetamine use, and, among users, females were significantly more likely to report smoking, while males were more likely to report injecting as the sole route of methamphetamine administration. RDS-adjusted estimates of prevalence of use by gender indicated that these findings held true after controlling for the sampling method [prevalence (95\% CI), males, 59\% (54-65); females, $75 \%(57-89)]$.

Among methamphetamine injectors, nearly $90 \%$ also reported injection of methamphetamine in combination with heroin; however, this proportion was higher among males compared to females $(93 \%$ vs. $84 \% ; p<0.01)$. Injection of 


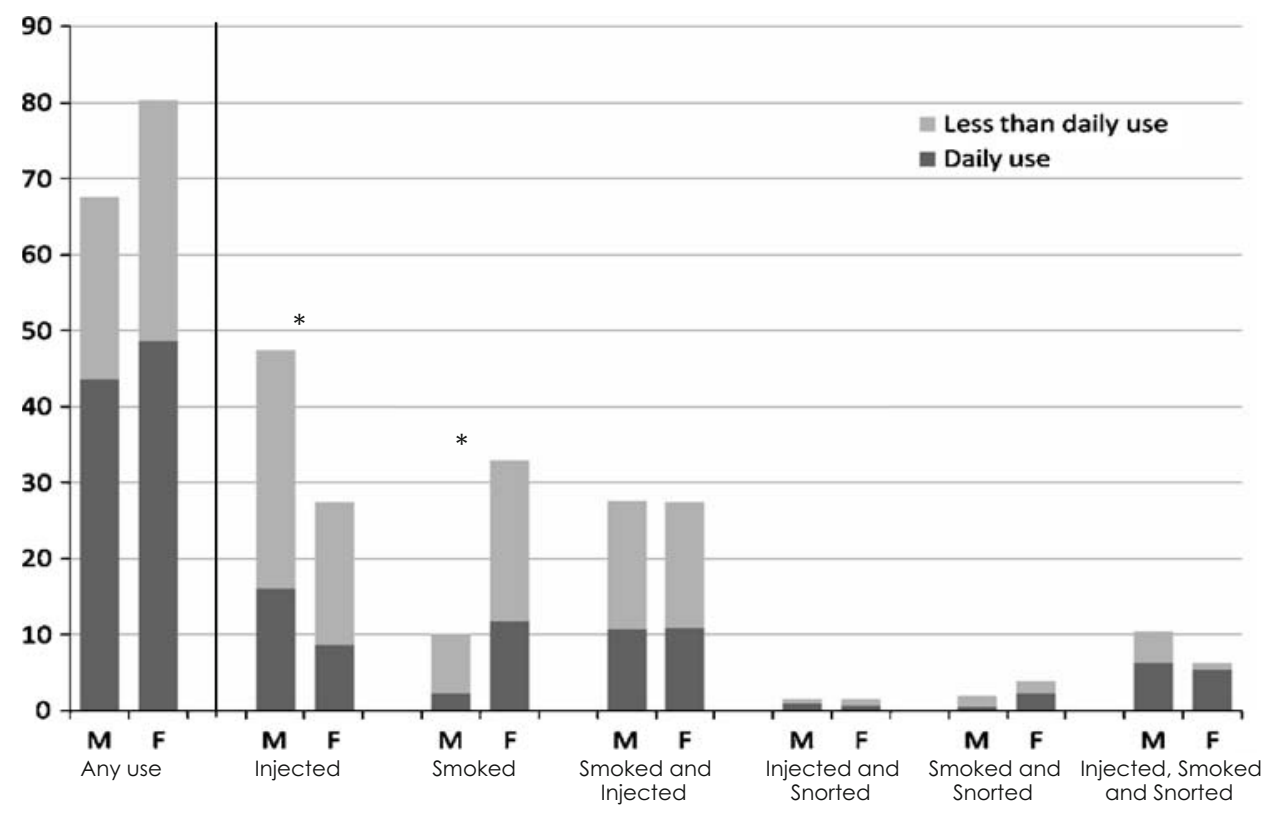

* significant difference in proportion reporting route of administration comparing males to females

FIGURE 1. Proportion of IDUs reporting any methamphetamine use and, among users, proportion reporting use by route of administration among male and female IDU in Tijuana, Mexico.

methamphetamine with cocaine was much less prevalent and did not differ by gender (males vs. females, $2.8 \%$ vs. $1.9 ; p=0.52$ ). Snorting was reported by similar proportions of male and female IDUs; however, only one individual reported snorting as the sole route of methamphetamine administration. Most individuals also reported smoking, injecting, or both. Regardless of gender, methamphetamine injection, either alone or in combination, was more likely to be reported on a daily basis compared to smoking and snorting. Daily use by other routes of administration did not differ by gender.

\section{Associations of Demographics and Sexual Risk with Methamphetamine Use by Gender}

Table 1 displays the associations of demographic and sexual behaviors with methamphetamine use, stratified by gender. Among males, younger age and reporting sexual activity with other males remained significantly associated with use of methamphetamine in the past 6 months when adjusting for variables significant in univariate analyses. Homelessness was only marginally significant in the final model. Among females, only age remained significantly associated with methamphetamine use. Of note, compared to their male counterparts, female methamphetamine users had higher levels of sexual risk and higher proportions of HIV and syphilis; however, only sex trade involvement had a significant interaction $(p=0.05)$ with gender in its association with methamphetamine use. There were no significant interactions between age and number of casual partners, sex work, or MSM.

Although MSM and WSW were defined as ever reporting same sex activity due to the small numbers reporting recent sexual activity ( $9 \%$ and $8 \%$, respectively), methamphetamine use among men and women reporting same sex activity in the 


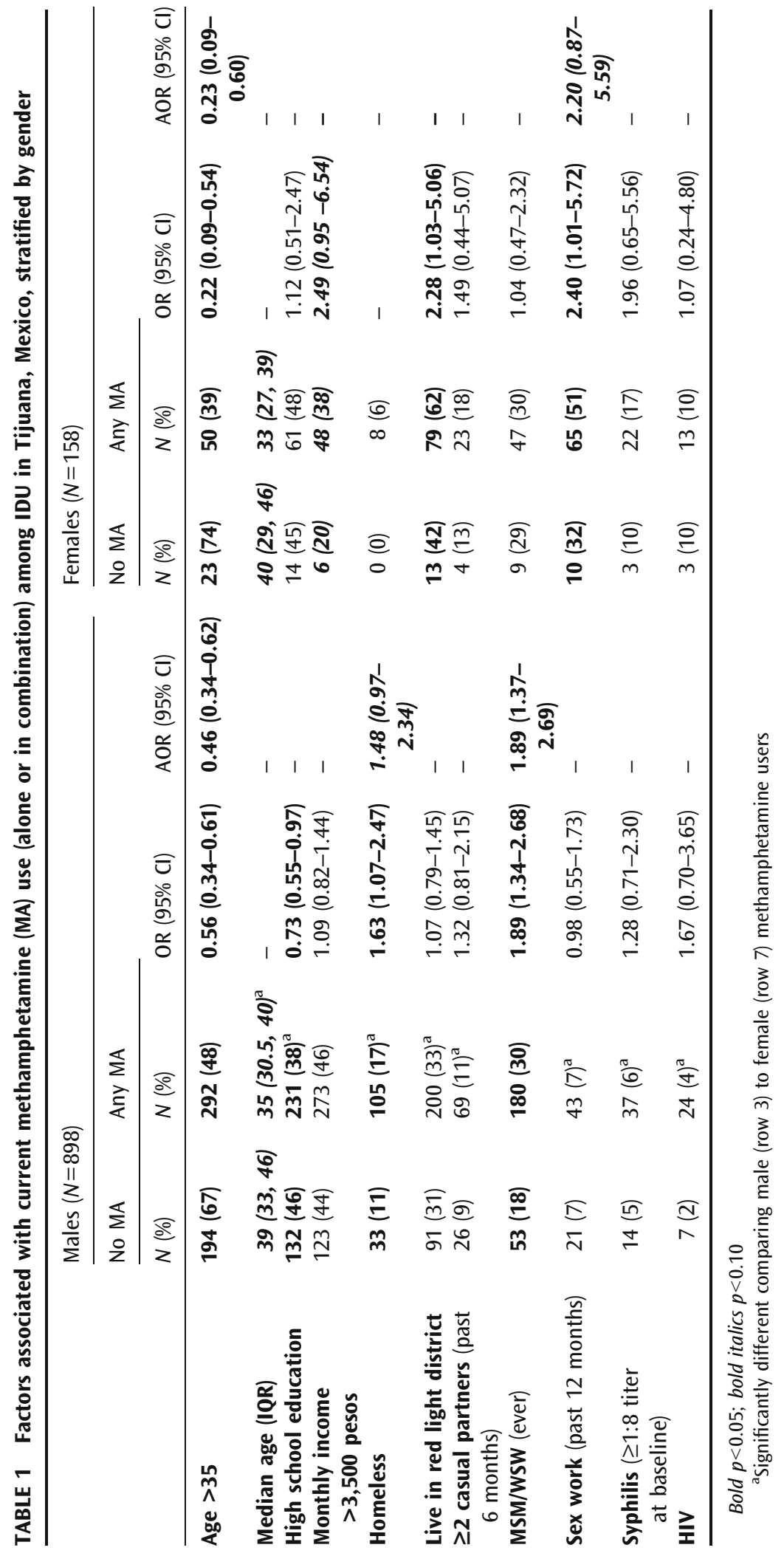


past 6 months were both high compared to those not reporting recent same sex activity ( $83 \%$ vs. $66 \%$ for MSM and $92 \%$ vs. $79 \%$ for WSW).

\section{Associations of Sexual Risk and Methamphetamine Use by Route of Administration and Gender}

Table 2 displays the factors associated with methamphetamine use by route of administration. Among males, reporting injection as the sole route of methamphetamine administration, whether alone or in combination with cocaine or heroin, was associated with younger age, being homeless, having sex with other men and testing HIV positive. Smoking methamphetamine was associated with lower education and was inversely associated with being homeless. Those that reported both injecting and smoking methamphetamine were significantly younger. Very few reported only snorting and injecting or only snorting and smoking; however, those reporting all three routes of administration were significantly younger, more likely to report $>2$ casual sex partners, having sex with other men, and sex work.

Table 3 shows the factors associated with specific routes of methamphetamine administration among females. Multiple stratifications by route of administration resulted in small sample sizes. However, similar to males, injection as the sole route of administration was associated with younger age, being homeless, and testing HIV positive at baseline. Smoking as the sole route of methamphetamine administration was associated with a higher income. Women reporting both smoking and injection of methamphetamine were more likely to report sex work. Very few women reported snorting methamphetamine (snorting and injecting, $N=2$; smoking and snorting, $N=5$; injecting, smoking, and snorting, $N=8$ ).

\section{DISCUSSION}

In our study of IDUs from Tijuana, we observed differences in the prevalence and routes of administration and the sociodemographic profile of methamphetamine use by gender. Although methamphetamine use was highly prevalent, over three quarters of females reported using methamphetamine in the last 6 months, compared to two thirds among males. Injection of methamphetamine, whether used alone or in combination with another substance, was more often reported by male IDUs, whereas smoking methamphetamine was more often reported by female IDU. While younger age was associated with methamphetamine use for both men and women, homelessness and history of homosexuality/bisexuality were associated with use among males, while sex work was associated with use among females. Among male IDU, specific sexual risk profiles emerged when exploring methamphetamine use by route of administration: reporting a history of MSM was associated with injection of methamphetamine, while more casual sex partners and recent sex work were uniquely associated with reporting multiple routes of methamphetamine administration.

While most studies of substance using populations indicate a higher prevalence of use among males, prevalence estimates of methamphetamine use in the USA indicate comparable rates by gender. ${ }^{3,14,15,36}$ In the present study, female IDUs in Tijuana had an even higher prevalence of use compared to males, a finding that has also been noted among male and female substance users entering drug treatment programs in Los Angeles. ${ }^{36}$ The higher prevalence of use among females may be driven in part by use among female sex workers-among IDU who reported never 


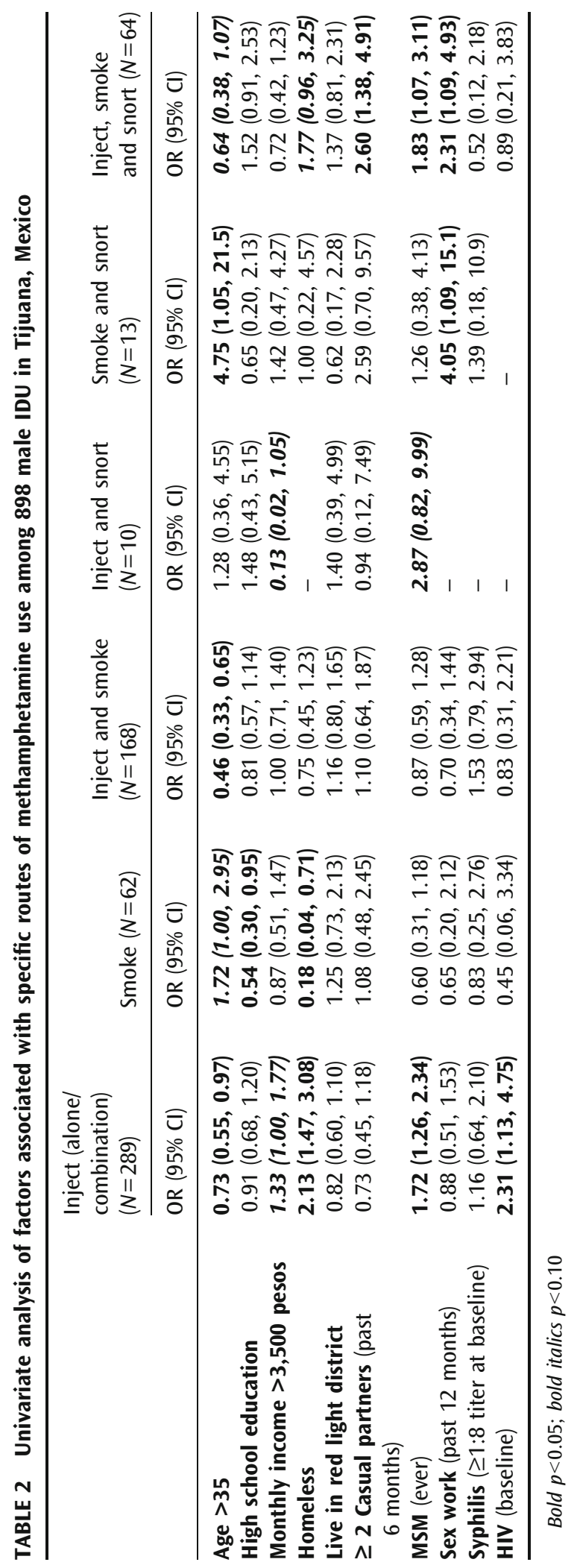


TABLE 3 Risk factors associated with specific routes of methamphetamine use among 158 female IDU in Tijuana, Mexico

\begin{tabular}{|c|c|c|c|}
\hline & $\begin{array}{l}\text { Inject (alone/ } \\
\text { combination) }(N=35)\end{array}$ & Smoke $(N=42)$ & $\begin{array}{l}\text { Inject and smoke } \\
(N=35)\end{array}$ \\
\hline & OR $(95 \% \mathrm{Cl})$ & OR $(95 \% \mathrm{Cl})$ & OR $(95 \% \mathrm{Cl})$ \\
\hline Age $>35$ & $0.38(0.17,0.86)$ & $0.95(0.47,1.93)$ & $0.84(0.39,1.79)$ \\
\hline High school education & $0.91(0.43,1.94)$ & $1.15(0.57,2.33)$ & $0.91(0.43,1.94)$ \\
\hline Monthly income $>3,500$ pesos & $0.58(0.25,1.34)$ & $2.58(1.24,5.37)$ & $1.03(0.46,2.28)$ \\
\hline Homeless & $6.67(1.51,29.5)$ & $0.38(0.05,3.18)$ & $0.49(0.06,4.10)$ \\
\hline Live in red light district & $1.76(0.80,3.92)$ & $0.94(0.46,1.92)$ & $1.50(0.69,3.28)$ \\
\hline $\begin{array}{l}\geq 2 \text { casual partners (past } \\
\quad 6 \text { months) }\end{array}$ & $0.56(0.18,1.75)$ & $1.48(0.61,3.62)$ & $2.02(0.81,5.01)$ \\
\hline WSW (ever) & $1.31(0.59,2.92)$ & $0.79(0.36,1.74)$ & $1.11(0.49,2.50)$ \\
\hline Sex work (past 12 months) & $0.91(0.43,1.94)$ & $1.15(0.57,2.33)$ & $2.62(1.19,5.73)$ \\
\hline Syphilis ( $\geq 1: 8$ titer at baseline) & $0.86(0.30,2.48)$ & $0.85(0.31,2.30)$ & $2.31(0.92,5.82)$ \\
\hline HIV (baseline) & $3.14(1.08,9.16)$ & - & $1.68(0.54,5.21)$ \\
\hline
\end{tabular}

Bold $p<0.05$; bold italics $p<0.10$

trading sex, females still have a higher prevalence of use compared to males although the difference is decreased and no longer significant.

The particularly high levels of methamphetamine use compared to other studies are noteworthy. In this population, daily methamphetamine injection was reported by nearly $20 \%$ of daily heroin injectors. While increases in methamphetamine use among injectors have been seen, the level of use tends to be lower, with one review from Australia estimating $10 \%$ of regular injectors reporting daily use of methamphetamine, regardless of route of administration. ${ }^{37,38}$ In a number of studies carried out to examine polydrug use and drug use trajectories over a 10-year cohort, methamphetamine use was common although used at much lower frequencies (average 1.5 days in past month). ${ }^{39}$ Despite no linear trends in use of other drugs over time, high-level heroin users showed higher levels of cocaine use in later years (4+ years injecting). ${ }^{39}$ It has also been reported that while earlier initiation of methamphetamine in the sequence of lifetime drug use was associated with experimental motivations (i.e. to have fun, to enhance sex), later initiation was associated with using methamphetamine as a substitution for other substances. ${ }^{40}$

Historically, availability and cost have been examined as predictors of expanded polydrug use or primary drug substitution. ${ }^{41-43}$ The impact of availability is clearly noted when comparing IDU in Tijuana to IDU in Ciudad Juarez, to the east. In Ciudad Juarez, heroin injectors reported very little methamphetamine use; instead, cocaine was the main secondary drug used along with heroin. ${ }^{32}$ In contrast, methamphetamine use has become increasingly common in Tijuana, which is situated on a major methamphetamine drug trafficking route. ${ }^{29}$ In-depth interviews among IDU in Tijuana pointed to the perceived cost-benefit of mixing heroin with cheaper substances, such as methamphetamine. ${ }^{32}$ These findings, combined with the higher availability of methamphetamine as opposed to cocaine in the Tijuana region, could explain the higher levels of polydrug use in our population, as the majority were long-term injectors.

The differences we observed in the routes of methamphetamine administration by gender may be explained by motivations for use. Studies examining motivations 
for methamphetamine use have found sexual motivations to be highly prevalent among both males and females. ${ }^{13,18,20}$ Qualitative research among both MSM and heterosexual populations has highlighted methamphetamine use as a way to facilitate sexual encounters, avoid unpleasant emotions or physical discomfort, and to increase libido. ${ }^{44-47}$ However, female methamphetamine users may have additional motivations for use of this drug, including the desire to lose weight, and to increase energy. ${ }^{7}$ In a study from San Diego, CA, methamphetamine use among females was strongly tied to depression, self-esteem, social stigma, and levels of use in social networks. ${ }^{24}$ An earlier study of female sex workers in Tijuana, Mexico indicated methamphetamine was used by the women to stay awake during work hours, ${ }^{48}$ supporting our finding that methamphetamine was more commonly used among FSWs. The association of methamphetamine use and sex work among females has been seen in other populations as well. ${ }^{49}$

The association observed between reporting a history of MSM and methamphetamine use was expected, as sexual motivations and sexualized contexts are more often reported among MSM. Methamphetamine use has been commonly reported to occur during circuit parties, at bathhouses, sex clubs, and for 'marathon sex' encounters. ${ }^{19,50-52}$ A qualitative study of substance using MSM in San Francisco found that the majority reported using cocaine in social party settings, and methamphetamine in sexual settings. ${ }^{18}$ Although unprotected intercourse was not examined in this analysis due to the low number of participants reporting recent sexual activity, other studies have found methamphetamine to be associated with increased unprotected anal intercourse among MSM, ${ }^{5,53,54}$ highlighting the potential importance of methamphetamine use in sexual contexts and increased risk of HIV/STI transmission.

Less surprising than the high prevalence of use among females compared to males was the observation that injection was a more common route of administration among males, while smoking was most common among females. Again, this is not unlike observations of the patterns of crack cocaine use in the USA, which have pointed to high rates of smoking among female sex workers. ${ }^{13,26,55}$ The finding that females are more likely to report smoking while males are more likely to report injection of methamphetamine concurs with other research among methamphetamine using populations. ${ }^{25}$ When comparing routes of methamphetamine administration by gender, it is important to note that the majority of female and male IDUs were daily injectors of some substance-typically heroin-suggesting that while males may be using methamphetamine in the context of their regular injection routine, females may be using in separate scenarios, such as prior to sex work as discussed above.

Although only a small proportion reported snorting methamphetamine, this route of administration was only reported in conjunction with smoking and/or injecting. Multiple routes of administration was associated with more potentially risky sexual behaviors, including a history of MSM, recent sex trade, and more casual partners and is consistent with previous studies examining non-injection methamphetamine use and sexual risk. ${ }^{4,10,56}$ As snorting methamphetamine was typically reported at lower frequencies, this route may indicate specific use in sexual settings (i.e. with MSM partners and sex trade clients) where injecting may not be the norm for the sexual partner.

Although we did not observe an association between methamphetamine use and HIV infection, overall, methamphetamine injection was significantly associated with HIV. This may be related to the increased frequency of injection and injection- 
related risks of HIV transmission or it may reflect exposure to higher risk injection networks. Despite that fact the other routes of methamphetamine administration were not associated with HIV, associations were seen with greater numbers of casual partners, sex work, and a history of MSM, suggesting that there is the potential for increased transmission among this subgroup. Prospective studies may provide better estimates of risk associated with specific combinations of substance use and routes of administration that were not able to be disentangled in this analysis.

There are several limitations to this study. The relatively low number of male participants who reported recent sexual activity, and the low number of female participants overall, precluded more sophisticated analyses of sexual partner types and condom use. Lower levels of recent sexual activity are not wholly surprising in an older cohort of mainly heroin using IDU; however, the high prevalence of methamphetamine use, which is typically associated with sexual activity, does raise questions about the generalizability of these findings. However, it is possible that the male IDUs in this study were not primarily using methamphetamine to seek sex partners, as some US studies of MSM suggest, ${ }^{19,57}$ but for other reasons that warrant exploration in future studies. As this analysis was based on baseline data, the associations are not causal, but do indicate subgroups of the substance using population which may merit more specific attentions. Finally, despite efforts to increase the female sample size through RDS methods, the overall number of female participants remained low and did not allow sufficient power to detect significant differences in the demographics and behaviors associated with specific routes of methamphetamine administration. It was known from previous qualitative work that, with respect to buying and using drugs, women tended to stay closer to home, often buying from the same person and using with friends in their own neighborhood. ${ }^{58}$ This differed from the men who often reported a range of drug sources and drug using locations, including 'picaderos' (shooting galleries) and other public locations. ${ }^{58}$ Thus, while the study site was centrally located and outreach was used to enhance enrollment and follow-up, it may be that women were less likely to venture out of their own areas to enroll in the study, even if they received RDS coupons.

Geographically speaking, the sample of IDU recruited, while concentrated in and around the Zona Roja, the 'red light' district, was still representative of IDU in Tijuana. The Zona Roja is well known to be an area with high levels of drug use, and therefore the concentration of participants from this area was not surprising. However, in total, participants represented 147 different defined neighborhoods (approximately 20\%) from areas all across Tijuana.

Methamphetamine use in this cohort of IDUs from Tijuana, Mexico is highly prevalent, and routes of administration were shown to differ significantly by gender. In a cohort of regular injectors, both injection and sexual risk for HIV must be addressed. Methamphetamine, which has often been linked to sexual environments and increased risk behaviors, was found to be associated with MSM, and, when snorted, with increased casual partners and recent sex work among male IDUs. The potential for increased unprotected sexual activity among methamphetamine using male IDUs is therefore present and regardless of self-identified sexual orientation, safe sex messages regarding both male and female sex partners and clients may be important to disseminate among male IDUs. Among females, smoking methamphetamine was the most prevalent form of use and was associated with sex work, potentially motivated by a need to maintain energy through a work shift. While not directly linked to sexual motivations, prolonged use and dependence may increase FSWs risk behavior with clients. While substance use in general may be seen as a factor contributing to HIV 
and STI risk, different substances may be used in different contexts and with different motivations. Analysis of the specific drug combinations and routes of administration may help to shed light on the situations in which decisions that affect an individual's risk are made. Although this cohort was primarily a heroininjecting cohort, the gender-specific routes of methamphetamine administration reported in this paper suggest that understanding these differences is critical to the design of future interventions. Further investigation of the context of methamphetamine use among gender-specific subgroups is therefore warranted to direct the implementation of appropriate interventions among male and female IDU.

\section{SUPPORT}

This work was supported by NIDA (Grant \#R01DA019829 and Award \#K99DA024135), the Canadian Institute for Health Research and the California HIV Research Program. Dr. Pollini is funded by a NIDA Mentored Research Scientist Development Award (K01DA022923).

OPEN ACCESS This article is distributed under the terms of the Creative Commons Attribution Noncommercial License which permits any noncommercial use, distribution, and reproduction in any medium, provided the original author(s) and source are credited.

\section{REFERENCES}

1. United Nations Office on Drugs and Crime. Amphetamines and Ecstasy: 2008 Global ATS Assessment. Vienna, Austria; 2008

2. McKetin R, Kozel N, Douglas J, et al. The rise of methamphetamine in Southeast and East Asia. Drug Alcohol Rev. 2008; 27(3): 220-228. doi:10.1080/09595230801923710.

3. Maxwell JC, Rutkowski BA. The prevalence of methamphetamine and amphetamine abuse in North America: a review of the indicators, 1992-2007. Drug Alcohol Rev. 2008; 27(3): 229-235. doi:10.1080/09595230801919460.

4. Schwarcz S, Scheer S, McFarland W, et al. Prevalence of HIV infection and predictors of high-transmission sexual risk behaviors among men who have sex with men. Am J Public Health. 2007; 97(6): 1067-1075. doi:10.2105/AJPH.2005.072249.

5. Carey JW, Mejia R, Bingham T, et al. Drug Use, High-Risk Sex Behaviors, and Increased Risk for Recent HIV Infection among Men who Have Sex with Men in Chicago and Los Angeles [published online ahead of print May 23, 2008]. AIDS Behav. 2008.

6. Zule WA, Costenbader EC, Meyer WJ Jr, Wechsberg WM. Methamphetamine use and risky sexual behaviors during heterosexual encounters. Sex Transm Dis. 2007; 34(9): 689-94. doi:10.1097/01.olq.0000260949.35304.22.

7. Semple SJ, Grant I, Patterson TL. Female methamphetamine users: social characteristics and sexual risk behavior. Women Health. 2004; 40(3): 35-50. doi:10.1300/J013v40n03_03.

8. Inglez-Dias A, Hahn JA, Lum PJ, Evans J, Davidson P, Page-Shafer K. Trends in methamphetamine use in young injection drug users in San Francisco from 1998 to 2004: the UFO Study. Drug Alcohol Rev. 2008; 27(3): 286-291. doi:10.1080/ 09595230801914784.

9. Molitor F, Ruiz JD, Flynn N, Mikanda JN, Sun RK, Anderson R. Methamphetamine use and sexual and injection risk behaviors among out-of-treatment injection drug users. Am J Drug Alcohol Abuse. 1999; 25(3): 475-493. doi:10.1081/ADA-100101874.

10. Lorvick J, Martinez A, Gee L, Kral AH. Sexual and injection risk among women who inject methamphetamine in San Francisco. J Urban Health. 2006; 83(3): 497-505. doi:10.1007/s11524-006-9039-4. 
11. Dluzen DE, Liu B. Gender differences in methamphetamine use and responses: a review. Gend Med. 2008; 5(1): 24-35. doi:10.1016/S1550-8579(08)80005-8.

12. Brecht ML, von O'Brien A, MC AMD. Methamphetamine use behaviors and gender differences. Addict Behav. 2004; 29(1): 89-106. doi:10.1016/S0306-4603(03)00082-0.

13. Rawson RA, Washton A, Domier CP, Reiber C. Drugs and sexual effects: role of drug type and gender. J Subst Abuse Treat. 2002; 22(2): 103-108. doi:10.1016/S0740-5472(01)00215-X.

14. Brady KT, Randall CL. Gender differences in substance use disorders. Psychiatr Clin North Am. 1999; 22(2): 241-252. doi:10.1016/S0193-953X(05)70074-5.

15. Cohen JB, Greenberg R, Uri J, Halpin M, Zweben JE. Women with methamphetamine dependence: research on etiology and treatment. J Psychoactive Drugs. 2007; Suppl 4: 347-51.

16. Brown AH, Domier CP, Rawson RA. Stimulants, Sex, and Gender. Sexual Addiction \& Compulsivity. 2005; 12(2): 169-180. doi:10.1080/10720160500203674.

17. Brewer DD, Golden MR, Handsfield HH. Unsafe sexual behavior and correlates of risk in a probability sample of men who have sex with men in the era of highly active antiretroviral therapy. Sex Transm Dis. 2006; 33(4): 250-255.

18. Nemoto T, Operario D, Soma T. Risk behaviors of filipino methamphetamine users in San Francisco: implications for prevention and treatment of drug use and HIV. Public Health Rep. 2002; 117(Suppl 1): S30-S38.

19. Semple SJ, Zians J, Strathdee SA, Patterson TL. Sexual marathons and methamphetamine use among HIV-positive men who have sex with men [published online ahead of print January 10, 2008]. Arch Sex Behav. 2008.

20. Diaz RM, Heckert AL, Sanchez J. Reasons for stimulant use among Latino gay men in San Francisco: a comparison between methamphetamine and cocaine users. J Urban Health. 2005; 82(Suppl 11): i71-i78. doi:doi:10.1093/jurban/jti026.

21. Parsons JT, Kelly BC, Wells BE. Differences in club drug use between heterosexual and lesbian/bisexual females. Addict Behav. 2006; 31(12): 2344-2349. doi:10.1016/j. addbeh.2006.03.006.

22. McCabe SE, Hughes TL, Boyd CJ. Substance use and misuse: are bisexual women at greater risk? J Psychoact Drugs. 2004; 36(2): 217-225.

23. Tucker JS, Ellickson PL, Klein DJ. Understanding differences in substance use among bisexual and heterosexual young women. Womens Health Issues. 2008; 18(5): 387-398. doi:10.1016/j.whi.2008.04.004.

24. Semple SJ, Zians J, Strathdee SA, Patterson TL. Psychosocial and behavioral correlates of depressed mood among female methamphetamine users. J Psychoact Drugs. 2007 November; Suppl 4: 353-66.

25. McKetin R, Ross J, Kelly E, et al. Characteristics and harms associated with injecting versus smoking methamphetamine among methamphetamine treatment entrants. Drug Alcohol Rev. 2008; 27(3): 277-285. doi:10.1080/09595230801919486.

26. McKetin R, Kelly E, McLaren J. The relationship between crystalline methamphetamine use and methamphetamine dependence. Drug Alcohol Depend. 2006; 85(3): 198-204. doi:10.1016/j.drugalcdep.2006.04.007.

27. Domier CP, Simon SL, Rawson RA, Huber A, Ling W. A comparison of injecting and noninjecting methamphetamine users. J Psychoact Drugs. 2000; 32(2): 229-232.

28. Matsumoto T, Kamijo A, Miyakawa T, et al. Methamphetamine in Japan: the consequences of methamphetamine abuse as a function of route of administration. Addiction. 2002; 97(7): 809-817. doi:10.1046/j.1360-0443.2002.00143.x.

29. Brouwer KC, Case P, Ramos R, et al. Trends in Production, Trafficking, and Consumption of Methamphetamine and Cocaine in Mexico. Subst Use Misuse. 2006; 41(5): 707-727. doi:10.1080/10826080500411478.

30. Bucardo J, Brouwer KC, Magis-RodrØguez C, et al. Historical trends in the production and consumption of illicit drugs in Mexico: implications for the prevention of blood borne infections. Drug Alcohol Depend. 2005; 79(3): 281-293. doi:10.1016/j.drugalcdep.2005.02.003. 
31. Cunningham JK, Liu LM, Muramoto M. Methamphetamine suppression and route of administration: precursor regulation impacts on snorting, smoking, swallowing and injecting [published online ahead of print April 16, 2008]. Addiction. 2008.

32. Case P, Ramos R, Brouwer K, et al. At the borders, on the edge: use of Injected methamphetamine in Tijuana and Ciudad Juarez, Mexico. J Immigr Minor Health. 2008; 10(1): 23-33.

33. Strathdee SA, Lozada R, Pollini RA, et al. Individual, social, and environmental influences associated with HIV infection among injection drug users in Tijuana, Mexico. J Acquir Immune Defic Syndr. 2008; 47(3): 369-376. doi:10.1097/QAI.0b013e318160d5ae.

34. Heckathorn DD. Respondent-driven sampling: a new approach to the study of hidden populations. Soc Probl. 1997; 44(2): 174-199. doi:10.1525/sp.1997.44.2.03x0221m.

35. Respondent-Driven Sampling Analysis Tool (RDSAT). Computer program. Version 5.6. Ithaca, NY: Cornell University; 2007.

36. Crevecoeur D, Rutkowski B, Rawson RA. The rise in treatment admissions for methamphetamine use in Los Angeles County from 2001 through 2005. J Psychoactive Drugs. 2007 November; Suppl 4: 383-92.:383-92.

37. Degenhardt L, Roxburgh A, Black E, et al. The epidemiology of methamphetamine use and harm in Australia. Drug Alcohol Rev. 2008; 27(3): 243-252. doi:10.1080/ 09595230801950572.

38. Fairbairn N, Kerr T, Buxton JA, Li K, Montaner JS, Wood E. Increasing use and associated harms of crystal methamphetamine injection in a Canadian setting. Drug Alcohol Depend. 2007; 88(2-3): 313-316. doi:10.1016/j.drugalcdep.2006.10.019.

39. Brecht ML, Huang D, Evans E, Hser YI. Polydrug use and implications for longitudinal research: ten-year trajectories for heroin, cocaine, and methamphetamine users. Drug Alcohol Depend. 2008; 96(3): 193-201. doi:10.1016/j.drugalcdep.2008.01.021.

40. Brecht ML, Greenwell L, Douglas Anglin M. Substance use pathways to methamphetamine use among treated users. Addict Behav. 2007; 32: 24-38. doi:10.1016/j. addbeh.2006.03.017.

41. Roxburgh A, Degenhardt L, Breen C. Changes in patterns of drug use among injecting drug users following changes in the availability of heroin in New South Wales, Australia. Drug Alcohol Rev. 2004; 23(3): 287-294. doi:10.1080/09595230412331289446.

42. Maher L, Li J, Jalaludin B, et al. Impact of a reduction in heroin availability on patterns of drug use, risk behaviour and incidence of hepatitis $C$ virus infection in injecting drug users in New South Wales, Australia. Drug Alcohol Depend. 2007; 89(2-3): 244-250. doi:10.1016/j.drugalcdep.2007.01.001.

43. Petry NM, Bickel WK. Polydrug abuse in heroin addicts: a behavioral economic analysis. Addiction. 1998; 93(3): 321-335. doi:10.1046/j.1360-0443.1998.9333212.x.

44. Halkitis PN, Mukherjee PP, Palamar JJ. Multi-level modeling to explain methamphetamine use among gay and bisexual men. Addiction. 2007; 102(Suppl 1): 76-83. 76-83.

45. Sherman SG, Gann D, German D, et al. A qualitative study of sexual behaviours among methamphetamine users in Chiang Mai, Thailand: a typology of risk. Drug Alcohol Rev. 2008; 27(3): 263-269. doi:10.1080/09595230801956520.

46. Mimiaga MJ, Fair AD, Mayer KH, et al. Experiences and Sexual Behaviors of HIVInfected MSM Who Acquired HIV in the Context of Crystal Methamphetamine Use. AIDS Educ Prev. 2008; 20(1): 30-41. doi:10.1521/aeap.2008.20.1.30.

47. Zule WA, Costenbader E, Coomes CM, et al. Stimulant use and sexual risk behaviors for HIV in rural North Carolina. J Rural Health. 2007; 23(Suppl): 73-78.

48. Bucardo J, Semple S, Fraga-Vallejo M, Davila W, Patterson T. A Qualitative Exploration of Female Sex Work in Tijuana, Mexico. Arch Sex Behav. 2004; 33(4): 343-351. doi:10.1023/B:ASEB.0000028887.96873.f3.

49. Weiser SD, Dilworth SE, Neilands TB, Cohen J, Bangsberg DR, Riley ED. Gender-specific correlates of sex trade among homeless and marginally housed individuals in San Francisco. J Urban Health. 2006; 83(4): 736-740. doi:10.1007/s11524-005-9019-0. 
50. Garofalo R, Mustanski BS, McKirnan DJ, Herrick A, Donenberg GR. Methamphetamine and young men who have sex with men: understanding patterns and correlates of use and the association with HIV-related sexual risk. Arch Pediatr Adolesc Med. 2007; 161(6): 591-596. doi:10.1001/archpedi.161.6.591.

51. Lee SJ, Galanter M, Dermatis H, McDowell D. Circuit parties and patterns of drug use in a subset of gay men. J Addict Dis. 2003; 22(4): 47-60. doi:10.1300/J069v22n04_05.

52. Mattison AM, Ross MW, Wolfson T, Franklin D. Circuit party attendance, club drug use, and unsafe sex in gay men. J Subst Abuse. 2001; 13(1-2): 119-126. doi:10.1016/S08993289(01)00060-8.

53. Fernandez MI, Bowen GS, Warren JC, et al. Crystal methamphetamine: a source of added sexual risk for Hispanic men who have sex with men? Drug Alcohol Depend. 2007; 86 (2-3): 245-252. doi:10.1016/j.drugalcdep.2006.06.016.

54. Rusch M, Lampinen T, Schilder A, Hogg RS. Unprotected Anal Intercourse Associated With Recreational Drug Use Among Young Men Who Have Sex With Men Depends on Partner Type and Intercourse Role. Sex Transm Dis. 2004; 31(8): 492-498. doi:10.1097/ 01.olq.0000135991.21755.18. Article.

55. Lejuez CW, Bornovalova MA, Reynolds EK, Daughters SB, Curtin JJ. Risk factors in the relationship between gender and crack/cocaine. Exp Clin Psychopharmacol. 2007; 15(2): 165-175. doi:10.1037/1064-1297.15.2.165.

56. Taylor MM, Aynalem G, Smith LV, Montoya J, Kerndt P. Methamphetamine use and sexual risk behaviours among men who have sex with men diagnosed with early syphilis in Los Angeles County. Int J STD AIDS. 2007; 18(2): 93-97. doi:10.1258/ 095646207779949709.

57. Patterson TL, Semple SJ, Zians JK, Strathdee SA. Methamphetamine-using HIV-positive men who have sex with men: correlates of polydrug use. J Urban Health. 2005; 82(Suppl 11): 120-126. doi:10.1093/jurban/jti031.

58. Cruz M, Mantsios A, Ramos R, et al. A Qualitative Exploration of Gender in the Context of Injection Drug Use in Two US-Mexico Border Cities. AIDS Behav. 2007; 11(2): 253262. doi:10.1007/s10461-006-9148-9. 